\title{
A superior drug carrier - aponeocarzinostatin in partially unfolded state fully protects the labile antitumor enediyne Aranganathan Shanmuganathan ${ }^{1,2}$, Thallapuranam Krishnaswamy Suresh Kumar ${ }^{3}$, Chiy-Mey Huang1, Chin Yu*2 and Der-Hang Chin*1
}

\author{
Address: ${ }^{1}$ Department of Chemistry, National Chung Hsing University, Taichung, Taiwan 40227, Republic of China, ${ }^{2}$ Department of Chemistry, \\ National Tsing Hua University, Hsinchu, Taiwan 30013, Republic of China and ${ }^{3}$ Department of Chemistry and Biochemistry, University of \\ Arkansas, Fayetteville, Arkansas 72701, USA \\ Email: Aranganathan Shanmuganathan - arangsindian@yahoo.com; Thallapuranam Krishnaswamy Suresh Kumar - sthalla@uark.edu; Chiy- \\ Mey Huang - chiy_mey@yahoo.com.tw; Chin Yu* - cyu.nthu@gmail.com; Der-Hang Chin* - chdhchin@dragon.nchu.edu.tw \\ * Corresponding authors
}

\section{Published: 23 May 2009}

Journal of Biomedical Science 2009, 16:48 doi:10.1 186/1423-0127-16-48

This article is available from: http://www.jbiomedsci.com/content//6/1/48

(c) 2009 Shanmuganathan et al; licensee BioMed Central Ltd.

This is an Open Access article distributed under the terms of the Creative Commons Attribution License (http://creativecommons.org/licenses/by/2.0), which permits unrestricted use, distribution, and reproduction in any medium, provided the original work is properly cited.
Received: 18 February 2009

Accepted: 23 May 2009

\begin{abstract}
Background: Neocarzinostatin is a potent antitumor drug consisting of an enediyne chromophore and a protein carrier.

Methods: We characterized an intermediate in the equilibrium unfolding pathway of aponeocarzinostatin, using a variety of biophysical techniques including I-anilino-8-napthalene sulfonate binding studies, size-exclusion fast protein liquid chromatography, intrinsic tryptophan fluorescence, circular dichroism, and $1 \mathrm{H}-{ }^{15} \mathrm{~N}$ heteronuclear single quantum coherence spectroscopy.

Results: The partially unfolded protein is in molten globule-like state, in which $\sim 60 \%$ and $\sim 20 \%$ tertiary and secondary structure is disrupted respectively. Despite lacking a fully coordinated tertiary structure for assembling a functional binding cleft, the protein in molten globule-like state is still able to fully protect the labile chromophore. Titration of chromophore leads the partially denatured apoprotein to fold into its native state.
\end{abstract}

Conclusion: These findings bring insight into conserving mechanism of neocarzinostatin under harsh environment, where even the partially denatured apoprotein exhibits protective effect, confirming the superiority of the drug carrier.

\section{Background}

Neocarzinostatin (NCS) is the most studied member within the family of natural enediyne-based chromoproteins with potent anti-tumor activity $[1,2]$. Holoneocarzinostatin (holoNCS) drug consists of a biologically active chromophore (NCS-Chr) that is non-covalently bound to a carrier apoprotein (apoNCS). NCS-Chr is very labile and can be inactivated quickly when it is not associated with apoNCS $[1,3,4]$. To carry out the protection role, a regular drug carrier protein must fold properly to form a welldefined specific binding cleft before it can accommodate the ligand molecule. Here we report an interesting observation that apoNCS in its partially unfolded intermediate state is able to efficiently bind and protect the labile NCS- 
Chr. Elucidation of the protein folding with respect to chromophore protection could serve as a starting point for rational drug carrier design strategies.

ApoNCS is an all $\beta$-sheet protein ( $11 \mathrm{kDa})$ consisting of an antiparallel $\beta$-barrel and a $\beta$-sheet domain (Fig. 1, NCS model in an aqueous environment at $\mathrm{pH} 7$ [5]). Folding and unfolding pathways of this small all $\beta$-sheet protein have been an interesting topic recently studied by various methods [6-11]. Results from some studies show that the folding/unfolding of apoNCS may not follow a simple two-state model, instead, stable folding intermediates may be involved in the transition pathways [9-11]. Nonetheless, detailed characterization of stable apoNCS folding/unfolding intermediates has not been reported.

Structural characterization of intermediates that populate in the folding/unfolding process is crucial to understand the protein folding mechanism. Equilibrium and kinetic intermediates have been identified in the unfolding/ refolding reactions of several proteins [12-15]. The best studied intermediate is the molten globule (MG) state $[16,17]$. The MG states are believed to be general folding intermediates because they populate both in the equilib-

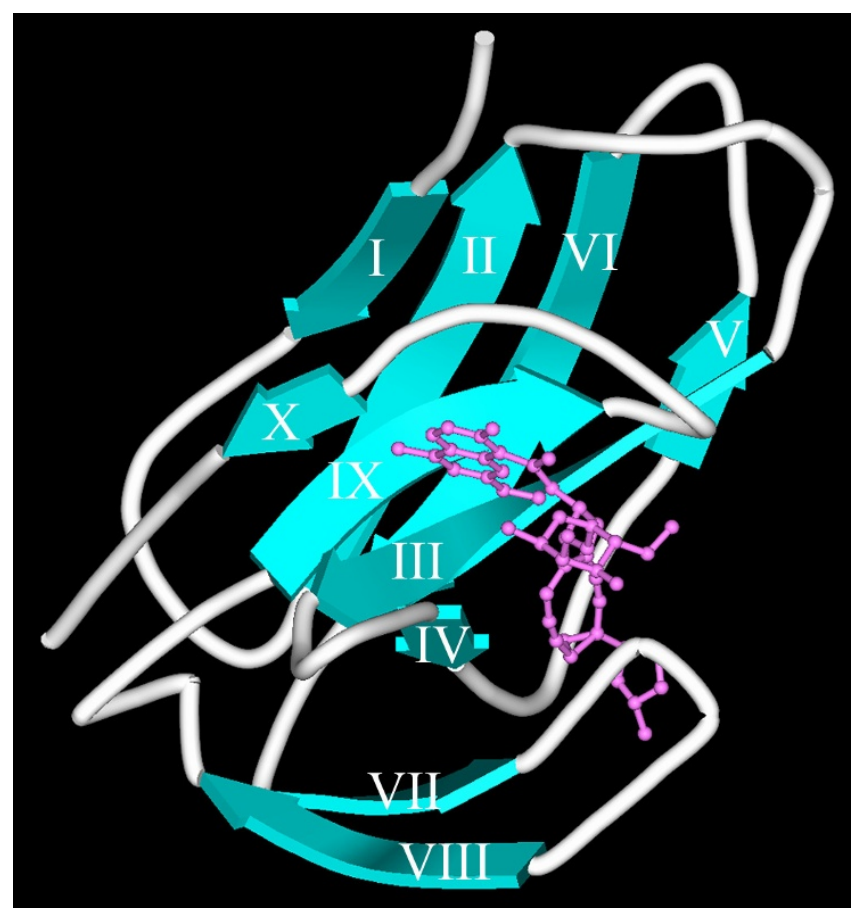

Figure I

Three-dimensional model of holoNCS in an aqueous environment at pH 7. The model was modified [5] from Brookhaven pdb. Inco.ent file. Chromophore is shown as a ball-and-stick representation (magenta). The all $\beta$-sheet holoNCS (cyan) consists of an antiparallel $\beta$-barrel and a small double-stranded $\beta$-sheet domain. rium and kinetic folding/unfolding pathways [16,17]. In the present study, we identified and characterized a stable intermediate in the guanidine hydrochloride ( $\mathrm{GdnHCl}$ )induced equilibrium unfolding pathway of apoNCS. Intermediate accumulates maximally in $1.2 \mathrm{M} \mathrm{GdnHCl}$ under acidic condition and has structural properties resembling that of a MG-like state. To gain insights into the biological role of apoNCS as a drug carrier, the interaction of MG-like intermediate with NCS-Chr was investigated.

\section{Methods \\ Materials}

NCS powder consisting apoNCS and NCS-Chr in a 1:1 molar ratio was a gift from Kayaku Co., Ltd., Itabashi-Ku, Tokyo, Japan. Extracted NCS-Chr was obtained after repeated methanol extractions of lyophilized NCS stock $\left(0.5 \mathrm{mM}\right.$, as determined by $\left.\varepsilon_{340}=10,800 \mathrm{M}^{-1} \mathrm{~cm}^{-1}\right)$ in 20 $\mathrm{mM}$ sodium citrate $(\mathrm{pH} 4)$, following the previously described method [18]. Extracted NCS-Chr was stored at $80^{\circ} \mathrm{C}$ in amber glass vials. Integrity and concentration of NCS-Chr were examined by UV spectroscopy and HPLC analysis. Labeled ${ }^{15} \mathrm{NH}_{4} \mathrm{Cl}$ and $\mathrm{D}_{2} \mathrm{O}$ were purchased from Cambridge Isotope Laboratories (Andover, MA, USA). All other chemicals used were of high quality analytical grade. All experiments were performed in $10 \mathrm{mM}$ phosphate buffer ( $\mathrm{pH} 3)$ at $25^{\circ} \mathrm{C}$.

\section{Expression and purification of apoNCS}

Recombinant apoNCS was overexpressed and purified by the procedure as described [19]. Homogeneity of the protein was examined by UV, HPLC and SDS-PAGE. Protein yield was about $2 \mathrm{mg} / \mathrm{L}$. Molecular mass of the purified protein was verified using ESI-Mass analysis.

\section{Preparation of isotope-enriched apoNCS}

Uniform ${ }^{15} \mathrm{~N}$ labeling was achieved by the established procedure [19]. E. coli BL21 Codon Plus strain (Stratagene, La Jolla, CA, USA) carrying the apoNCS gene was cultured in $\mathrm{M} 9$ minimal medium containing ${ }^{15} \mathrm{NH}_{4} \mathrm{Cl}$ supplemented with vitamin $B_{1}$. Final yield of the ${ }^{15} \mathrm{~N}$ labeled protein was about half of the corresponding unlabeled protein expressed in LB medium.

\section{Steady-State fluorescence measurements}

Fluorescence spectra were collected using a Hitachi F4500 spectrofluorimeter at a resolution of 2.5 or $10 \mathrm{~nm}$. For GdnHCl-induced unfolding study, excitation wavelength was set at $280 \mathrm{~nm}$. Intrinsic fluorescence measurements were made at $25^{\circ} \mathrm{C}$. Binding affinity of 1 -anilino-8napthalene sulfonate (ANS) to apoNCS at various concentrations of $\mathrm{GdnHCl}$ was monitored in a wavelength range of 375 to $625 \mathrm{~nm}$ using an excitation wavelength at 355 $\mathrm{nm}$. Excitation and emission bandwidths were set at 5 $\mathrm{nm}$. Concentration of ANS and protein was $100 \mu \mathrm{M}$ and 
$10 \mu \mathrm{M}$ respectively. All samples were prepared in $10 \mathrm{mM}$ phosphate buffer at $\mathrm{pH} 3$.

\section{Circular dichroism (CD) spectroscopy}

All CD measurements are carried out on a Jasco J-715 spectropolarimeter (Tokyo, Japan) equipped with a circulating water bath (Neslab, model RTE-140) (Portsmouth, $\mathrm{NH}$, USA). Measurements were made using a $0.1 \mathrm{~cm}$ pathlength water-jacketed quartz cell. Each spectrum represents an average of 30 scans with a scan speed of $50 \mathrm{~nm} /$ $\mathrm{min}$. Concentration of the protein used was $15 \mu \mathrm{M}$. Background corrections were made in all spectra. Bandwidth was set to $1 \mathrm{~nm}$ and all spectra were acquired at $25^{\circ} \mathrm{C}$.

\section{Size-exclusion chromatography (SEC)}

Gel-filtration experiments were carried out at $25^{\circ} \mathrm{C}$ on a superdex-100 column using a Pharmacia AKTA FPLC chromatographic device (GE Healthcare Bio-Sciences AB, Uppsala, Sweden). Column was equilibrated with 2 bed volumes of $10 \mathrm{mM}$ phosphate buffer (pH 3) containing appropriate concentrations of $\mathrm{GdnHCl}$ at a flow rate of 1 $\mathrm{ml} / \mathrm{min}$. Concentration of protein used for each analysis was approximately $250 \mu \mathrm{g} / \mathrm{ml}$ (dissolved in appropriate concentrations of $\mathrm{GdnHCl}$ ). Protein elution was monitored by UV absorbance at $280 \mathrm{~nm}$.

\section{Thermal denaturation experiments}

Thermal stability of apoNCS in $10 \mathrm{mM}$ phosphate buffer (pH 3) in the presence or absence of $1.2 \mathrm{M} \mathrm{GdnHCl}$ was monitored by far-UV CD at $224 \mathrm{~nm}$. Changes in ellipticity with temperature were followed from $5-91^{\circ} \mathrm{C}$ at an increment of $3^{\circ} \mathrm{C}$. Experiments were performed using a waterjacketed cell connected to a thermal circulator equipped with a microprocessor and temperature sensor. Protein sample $(25 \mu \mathrm{M})$ was allowed to equilibrate for 10 minutes at each temperature before data acquisition.

\section{NMR experiments}

The NMR experiments were carried out on a Bruker DMX $600 \mathrm{MHz}$ NMR spectrometer (Rheinstetten, Germany) at $25^{\circ} \mathrm{C}$. A $5 \mathrm{~mm}$ inverse probe with a self-shielded z-gradient was used to obtain all gradient-enhanced ${ }^{1} \mathrm{H}_{-15}{ }^{15} \mathrm{~N}$ HSQC spectra $[20,21]$. ${ }^{15} \mathrm{~N}$ decoupling during acquisitions was achieved using the GARP sequence [22]. Total of 2048 complex data points were collected in the ${ }^{1} \mathrm{H}$ dimension of the ${ }^{1} \mathrm{H}_{-15} \mathrm{~N}$ HSQC experiments. In the indirect ${ }^{15} \mathrm{~N}$-dimension spectra, 512 complex data points were collected. The HSQC spectra were recorded by 32 scans at all concentrations of $\mathrm{GdnHCl} .{ }^{15} \mathrm{~N}$ chemical shifts were referenced using consensus ratio of 0.0101329118 . All spectra were processed on a Silicon Graphics workstation using XWINNMR and Sparky softwares.

\section{Binding experiments}

Binding experiments were performed in $10 \mathrm{mM}$ phosphate buffer ( $\mathrm{pH} 3$ ) by incubating $20 \mu \mathrm{M}$ apoNCS (in the presence or absence of $1.2 \mathrm{M} \mathrm{GdnHCl}$ ) with NCS-Chr at $1: 1$ molar ratio for 30 minutes at $25^{\circ} \mathrm{C}$. The final methanol content introduced from NCS-Chr stock was kept minimal to about $4 \%(\mathrm{v} / \mathrm{v})$. Analyses of the proteinbound NCS-Chr after binding experiments were performed through a Waters $\mu$-Bondapak reverse phase $C_{18}$ column by a Waters Millennium HPLC equipped with a model 600E solvent delivery system, a 996 photodiode array detector and either a Waters 474 or a Jasco FP-1520 fluorescence detector following previously described method [23].

\section{Results \\ GdnHCl-induced unfolding of apoNCS does not follow a two-state model}

GdnHCl-induced equilibrium unfolding of apoNCS at pH 3 was monitored by changes in intrinsic tryptophan fluorescence and far-UV CD at $224 \mathrm{~nm}$. Circular dichroism spectrum of the all $\beta$-sheet apoNCS protein is somewhat atypical [24] with a large positive maximum at 224 $\mathrm{nm}$ and small negative minimum centered at $212 \mathrm{~nm}$ [7]. Fluorescence and far-UV CD spectral probes have been shown to reliably report the gross tertiary and secondary structural changes that possibly occur during the unfolding of apoNCS [11]. Fig. 2 shows that GdnHCl-induced equilibrium unfolding of apoNCS under acidic condition (pH 3) monitored by changes in intrinsic tryptophan fluorescence is completely reversible. The fluorescence unfolding profile of apoNCS shows that the protein starts to unfold in $0.6 \mathrm{M} \mathrm{GdnHCl}$. Unfolding of the protein is completed beyond $3 \mathrm{M} \mathrm{GdnHCl}$ (Fig. 2). $\mathrm{C}_{\mathrm{m}}$ (concentration of $\mathrm{GdnHCl}$ at which $50 \%$ of the protein molecules are in denatured state) and ' $m$ ' (measure of cooperativity of unfolding process) are $1.1 \pm 0.1 \mathrm{M}$ and $7.3 \pm 0.7 \mathrm{~kJ} \mathrm{~mol}^{-}$ ${ }^{1} \mathrm{M}^{-1}$ respectively. Change in free energy of unfolding obtained in the absence of the denaturant $\left[\Delta \mathrm{G}\left(\mathrm{H}_{2} \mathrm{O}\right)\right]$ is estimated to be $8.5 \pm 0.1 \mathrm{~kJ} \mathrm{~mol}^{-1}$. Interestingly, the Gdn$\mathrm{HCl}$-induced unfolding curve obtained by measuring ellipticity changes at $224 \mathrm{~nm}$ is not superimposable with that obtained by monitoring the changes in intrinsic tryptophan fluorescence. These results possibly suggest that the unfolding of apoNCS may not follow a two-state model, and may involve accumulation of stable intermediate states (Fig. 2). $\mathrm{C}_{\mathrm{m}^{\prime}}{ }^{\prime} m^{\prime}$ and $\left[\Delta \mathrm{G}\left(\mathrm{H}_{2} \mathrm{O}\right)\right]$ estimated from the unfolding profile obtained using far-UV CD are $1.8 \pm 0.1 \mathrm{M}, 4.4 \pm 0.2 \mathrm{~kJ} \mathrm{~mol}^{-1} \mathrm{M}^{-1}$ and $8.6 \pm 0.5 \mathrm{~kJ} \mathrm{~mol}^{-1}$ respectively.

\section{A stable equilibrium unfolding intermediate of apoNCS}

Size-exclusion chromatography (SEC) is a useful technique to acquire information on integral changes of molecular dimensions under the effect of the denaturant 


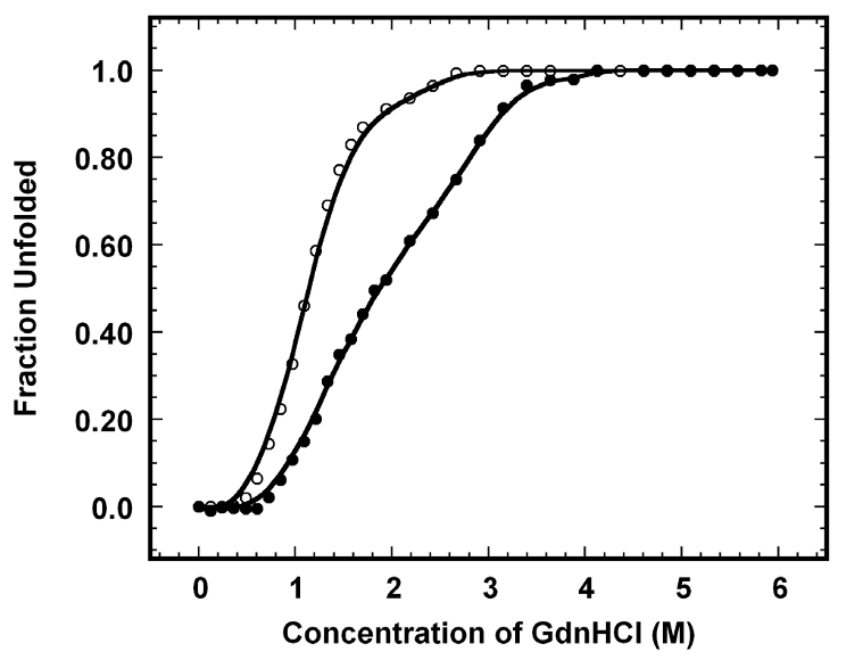

Figure 2

Fraction of unfolded apoNCS at various concentrations of $\mathbf{G d n H C l}$. The $\mathrm{GdnHCl}$-induced unfolding at $25^{\circ} \mathrm{C}$ was monitored by changes in fluorescence emission at 340 $\mathrm{nm}$ (excited at $280 \mathrm{~nm}$ ) (open circle) and ellipiticity at 224 $\mathrm{nm}$ (black circle). The concentration of apoNCS used in the fluorescence and far-UV CD experiments was 5 and I $5 \mu \mathrm{M}$, respectively. Samples were prepared in $10 \mathrm{mM}$ phosphate buffer at $\mathrm{pH} 3$.

$[25,26]$. This technique has been successfully used to identify and obtain hydrodynamic data on stable intermediates in the folding/unfolding pathway of proteins [25]. ApoNCS in $10 \mathrm{mM}$ phosphate buffer ( $\mathrm{pH} 3$ ) in the absence of $\mathrm{GdnHCl}$ elutes as a single peak with an elution volume of $\sim 129 \mathrm{ml}$ (Fig. 3). On increasing the concentration of $\mathrm{GdnHCl}$ from 0 to $0.2 \mathrm{M}$, the elution volume of the protein peak sharply decreases to about $\sim 111 \mathrm{~mL}$. Interestingly, elution volume of the protein does not significantly change when the $\mathrm{GdnHCl}$ concentration is increased from $0.4 \mathrm{M}$ to $1.2 \mathrm{M}$ (Fig. 3). A clear plateau can be observed in the plot of elution volume versus concentration of $\mathrm{GdnHCl}$ suggesting the accumulation of a stable equilibrium intermediate in this range of denaturant concentration. Beyond 1.2 M GdnHCl, elution volume of the protein changes drastically and profile eventually reaches a plateau at $2 \mathrm{M} \mathrm{GdnHCl}$. It should be mentioned that apoNCS elutes as a single peak at various concentrations of $\mathrm{GdnHCl}$ indicating that the protein exists as a single population at all concentrations of the denaturant (Fig. 3, inset). Uversky [26], using size-exclusion chromatography to study the denaturant-induced equilibrium unfolding profile of proteins, showed that proteins such as $\beta$-lactamase, bovine carbonic anhydrase and $\beta$-lactoglobulin, which denature through a MG-like intermediate, also elute as single peaks at all concentrations of the denatu- rant [26]. The results of the SEC-FPLC experiment clearly show that the GdnHCl-induced unfolding of apoNCS proceeds through the accumulation of a stable intermediate at around $1.2 \mathrm{M} \mathrm{GdnHCl}$ at $\mathrm{pH} 3$ (Fig. 3). At pH above 3 no stable intermediate was observed. The first phase of apoNCS unfolding between 0-1.2 M GdnHCl appears to represent an equilibrium transition between the native and the intermediate state.

\section{Partially unfolded apoNCS intermediate resembles MG- like state}

Under optimal conditions ( $\mathrm{pH} 3,1.2 \mathrm{M} \mathrm{GdnHCl)}$ where the stable unfolding intermediate of apoNCS accumulates, considerable percentage (about $80 \%$ ) of the secondary structure remains undisrupted, whereas $60 \%$ of the tertiary structure is disrupted (Fig. 2). This is a good indication that the stable intermediate could be in MG-like state. ANS is a fluorescent dye that binds to the hydrophobic regions of proteins [27] and this fluorescent probe has been immensely useful in the identification of equilibrium intermediates such as MG states. MG intermediates usually display significant exposure of hydrophobic cores to the solvent. Therefore, ANS binds strongly to MG states and fluoresces intensely $[28,29]$. The dye generally exhibits weak binding affinity to the native and denatured proteins [28]. The binding affinity of apoNCS to ANS at various concentrations of $\mathrm{GdnHCl}$ was monitored by changes in the emission intensity at $475 \mathrm{~nm}$ (Fig. 4). The ANS emission intensity upon binding to apoNCS in $1.2 \mathrm{M}$ $\mathrm{GdnHCl}$ is significantly higher than that observed with the protein in its folded state (Fig. 4). Further increase in the concentration of $\mathrm{GdnHCl}$ (beyond $1.2 \mathrm{M}$ ) results not only in the progressive decrease in the emission intensity but also in a continuous red shift in the emission maxima. The protein in its unfolded conformations, at and beyond $3 \mathrm{M} \mathrm{GdnHCl}$, exhibits weak binding to ANS (Fig. 4). Thus, the results of ANS binding and SEC-FPLC experiments, analyzed in conjunction, clearly suggest that a MG-like intermediate accumulates maximally in $1.2 \mathrm{M} \mathrm{GdnHCl}$.

\section{Thermal stability of MG-like intermediate of apoNCS}

It is important to understand the stability of the equilibrium unfolding intermediate states in relation to the folded state of the protein. In this context, we carried out thermal denaturation of apoNCS in the presence and absence of $1.2 \mathrm{M} \mathrm{GdnHCl}$ at $\mathrm{pH} 3$ (Fig. 5). Thermal unfolding monitored by ellipticity changes at $224 \mathrm{~nm}$ shows that apoNCS at $\mathrm{pH} 3$ unfolds to denatured state at temperatures beyond $62^{\circ} \mathrm{C}$ (Fig. 5). Apparent $\mathrm{T}_{\mathrm{m}}$ (temperature at which $50 \%$ of the protein molecules exist in the denatured state) of the unfolding reaction is about $52 \pm$ $1^{\circ} \mathrm{C}$. In marked contrast, the MG-like state of the protein at the same pH level in $1.2 \mathrm{M} \mathrm{GdnHCl}$ unfolds almost completely beyond $50^{\circ} \mathrm{C}$ (Fig. 5 ). $\mathrm{T}_{\mathrm{m}}$ of the MG-like state to denatured state of apoNCS is $35 \pm 1^{\circ} \mathrm{C}$. These results 


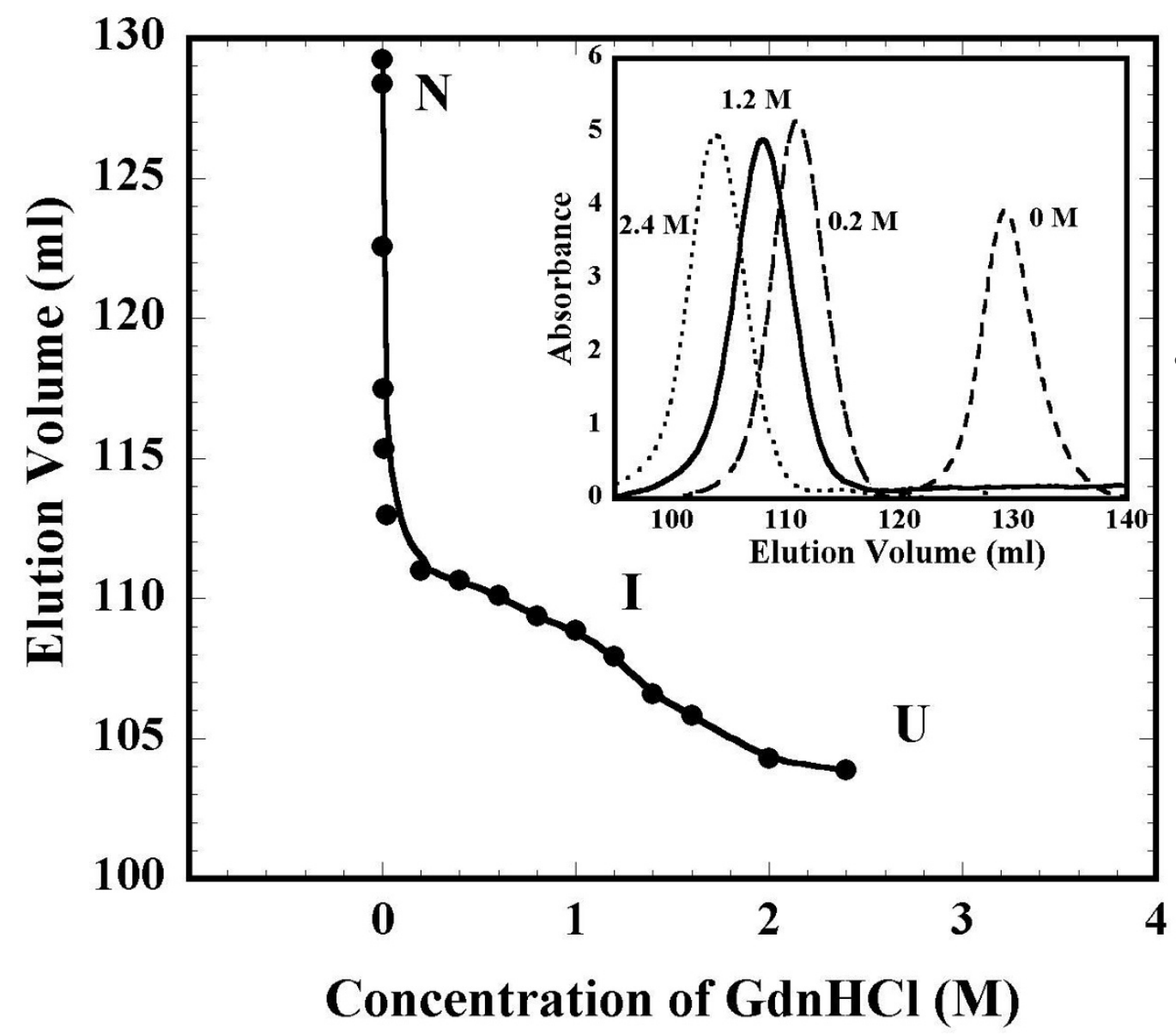

Elution profile of apoNCS at: $0 \mathrm{M}(---) ; 0.2 \mathrm{M}(\ldots) ; 1.2 \mathrm{M}(-)$; and $2.4 \mathrm{M}(\ldots \ldots)$ GdnHCl concentration.

Figure 3

GdnHCl-induced unfolding of apoNCS demonstrated by SEC analyses. The changes in elution volume of apoNCS at $\mathrm{pH} 3$ were measured at various concentration of $\mathrm{GdnHCl}$. Transition from native $(\mathrm{N})$ to intermediate $(\mathrm{I})$ state occurs in the first phase (0-I.2 M GdnHCl). Transition from intermediate (I) to denatured (D) state occurs in the second phase beyond I.3 $\mathrm{M} \mathrm{GdnHCl}$.

suggest that the protein in the MG-like state is significantly less stable than the folded state. It appears that some of the interactions stabilizing the compactly folded conformation are disrupted in MG-like state.

\section{NMR studies on structural changes in MG-like state of apoNCS}

Two-dimensional ${ }^{1} \mathrm{H}-{ }^{15} \mathrm{~N}$ heteronuclear single quantum coherence (HSQC) NMR spectroscopy serves as a fingerprint of the conformational state of a protein. It provides residue level information on the structural changes that possibly occur during unfolding/folding processes. We synthesized and purified ${ }^{15} \mathrm{~N}$-labeled apoNCS and monitored $\mathrm{GdnHCl}$-induced unfolding by ${ }^{1} \mathrm{H}-{ }^{15} \mathrm{~N}$ chemical shift perturbation. Heteronuclear correlation experiments have been shown to be very sensitive because of high magnetization transfer between directly bonded nuclei. Fig. $6 \mathrm{~A}$ shows that $2 \mathrm{D}{ }^{1} \mathrm{H}-{ }^{15} \mathrm{~N}$ HSQC spectrum of apoNCS at $\mathrm{pH} 3$ in the absence of $\mathrm{GdnHCl}$ is well-dispersed, which is a characteristic feature of a folded protein. Striking differences can be discerned in the HSQC spectra obtained in the absence and presence of $1.2 \mathrm{M} \mathrm{GdnHCl}$. Many cross peaks in HSQC spectrum of the protein in $1.2 \mathrm{M} \mathrm{GdnHCl}$ are broadened, suggesting the overall flexibility of residues in the intermediate state (in $1.2 \mathrm{M} \mathrm{GdnHCl}$ ) is significantly higher than that in the folded state (Fig. 6A). Though it is not easy to identify residues in the partially denatured state, we attempted to make an assignment by recording a series of HSQC NMR spectrum of apoNCS at $\mathrm{pH} 5$ to 3 with 0.5 decrement of $\mathrm{pH}$ level. ${ }^{1} \mathrm{H}$ and ${ }^{15} \mathrm{~N}$ 


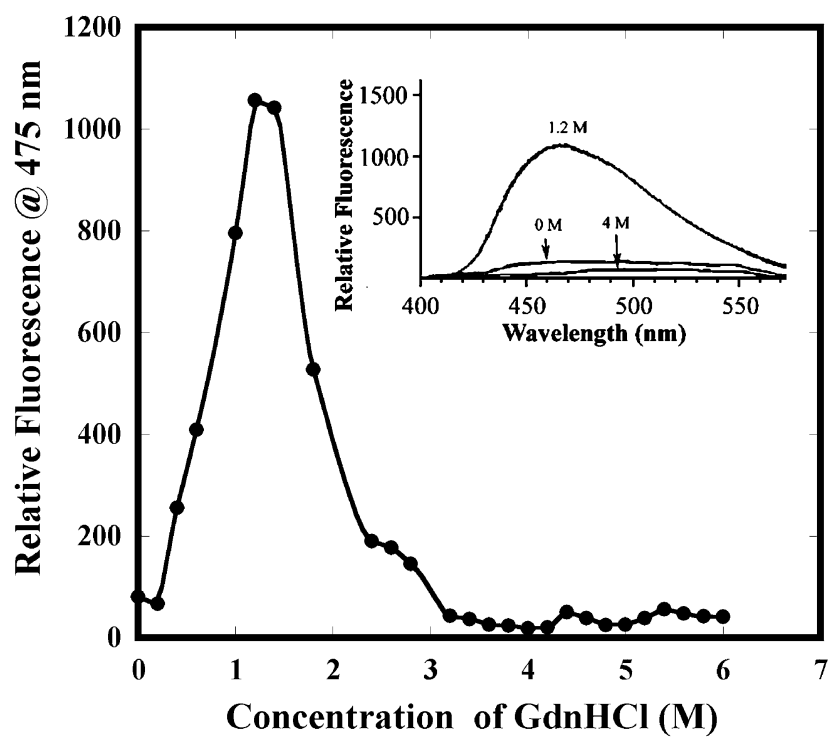

Figure 4

Binding of ANS to apoNCS at various concentrations of $\mathbf{G d n H C l}$. Fluorescent emission intensity of ANS (I00 $\mu \mathrm{M})$ in the presence of apoNCS $(10 \mu \mathrm{M})$ was followed at $25^{\circ} \mathrm{C}$ in different concentration of $\mathrm{GdnHCl}$ using an excitation wavelength at $355 \mathrm{~nm}$. Samples were prepared in $10 \mathrm{mM}$ phosphate buffer at $\mathrm{pH} 3$. Inset shows emission spectra of ANS with apoNCS in native $(\mathrm{N}$, in $0 \mathrm{M} \mathrm{GdnHCl})$, intermediate $(\mathrm{I}$, in $\mathrm{I} .2 \mathrm{M} \mathrm{GdnHCl})$ and denatured $(D$, in $4 \mathrm{M} \mathrm{GdnHCl})$ state.

backbone chemical shifts of native apoNCS at pH 5 were assigned based on the reported values (105 of 113 residues of apoNCS were reported) [30]. Once the cross peaks of apoNCS spectrum obtained at pH 3 were assigned, they were compared with those recorded at $\mathrm{pH} 3$ in the presence of $\mathrm{GdnHCl}$. By stepwise comparison, we identified 70 residues in the spectrum of MG-like state of apoNCS. The weighted average (of ${ }^{15} \mathrm{~N}$ and ${ }^{1} \mathrm{H}$ ) chemical shift perturbation $\left.\left((\Delta \delta)=\left[(\delta \mathrm{H})^{2}+0.2\left(\delta^{15} \mathrm{~N}\right)^{2}\right)\right]^{1 / 2}\right)$ of residues in MG-like state of apoNCS is shown in Fig. $6 \mathrm{~B}$, in which the unassigned residues (residues 1-3, 5, 8, 9, 15, 19, 24, 28, 31, 38-44, 46, 48, 49, 53, 54, 56, 59, 62, 66, 75, 78, 79, $81,82,87,89,92,93,97,99,100,103,105,106,112)$ as well as the residues with perturbation below $0.1 \mathrm{ppm}$ (the threshold of the average minimal perturbation) were eliminated. The result clearly shows that residues at $\mathrm{N}$ and C-termini of the protein are significantly perturbed in the MG-like state (Fig. 6B). Most of the residues that show significant chemical shift changes or peak broadening appear to be those not involved in the secondary structure formation (Fig. 6A and 6B). HSQC spectra of apoNCS acquired beyond $4 \mathrm{M}$ GdnHCl show significant decrease in chemical shift dispersion in ${ }^{1} \mathrm{H}$ dimension indicating complete unfolding of the protein (Fig. 6A). The results of the NMR experiments suggest that although the interac-

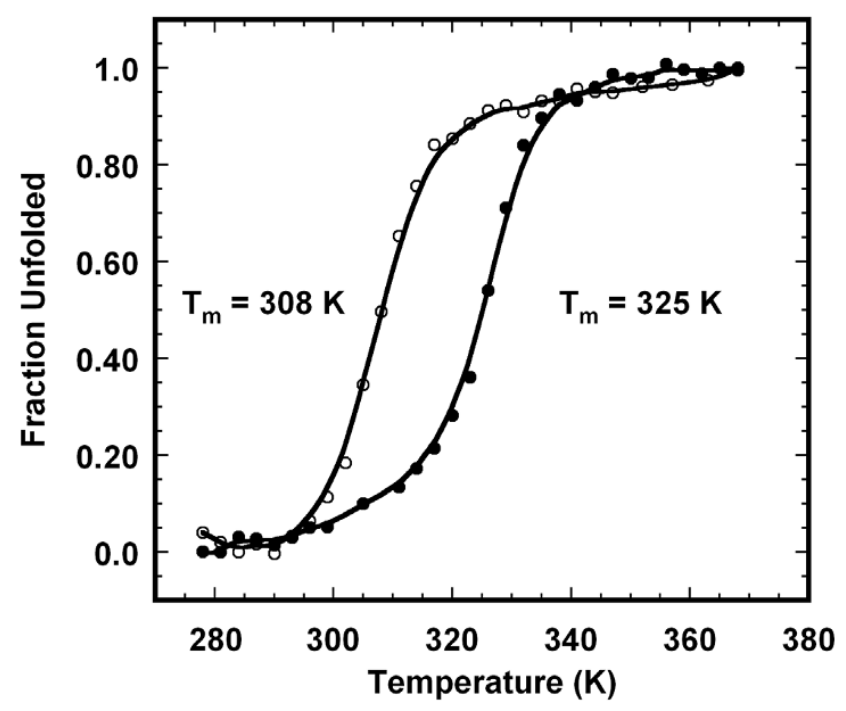

Figure 5

Thermal unfolding of apoNCS at the intermediate state. Heat-induced unfolding of apoNCS was monitored by changes in ellipticity at $224 \mathrm{~nm}$ in the absence (black circle) and presence (open circle) of I.2 M GdnHCl. ApoNCS samples $(25 \mu \mathrm{M})$ were prepared in $10 \mathrm{mM}$ phosphate buffer at $\mathrm{pH} 3$.

tions at loop regions and both $\mathrm{N}$ - and the C-termini of the protein are perturbed, the secondary structural elements in the protein appear to be mostly unaffected in the equilibrium intermediate in $1.2 \mathrm{M} \mathrm{GdnHCl}$.

\section{ApoNCS in partially unfolded MG-like state fully protects labile NCS-Chr}

NCS manifests its antineoplastic effects through damage to the cellular genome [1]. The very potent cytotoxic activities come from the enediyne warhead NCS-Chr, which is very labile by itself [3]. At pH 8, the life time of free NCSChr in an aqueous environment is only few seconds [4]. Binding with apoNCS increases the life time of NCS-Chr to several thousand folds [4]. Thus, the degree of protection against spontaneous NCS-Chr degradation can serve as a good probe to assess the binding affinity towards NCS-Chr [4,31]. To examine whether apoNCS in MG-like state binds to NCS-Chr, an aliquot of NCS-Chr was incubated with 1:1 molar ratio of apoNCS at pH 3 and $25^{\circ} \mathrm{C}$ for $30 \mathrm{~min}$ in the presence and absence of $1.2 \mathrm{M} \mathrm{GdnHCl}$. The remaining amount of intact NCS-Chr was analyzed by HPLC following the previously established method [23]. The results clearly show that NCS-Chr was fully protected in the presence of either folded or MG-like state of apoNCS (Table 1). Evidently, the MG-like state of apoNCS in $1.2 \mathrm{M} \mathrm{GdnHCl}$ retains its full ability to bind to its enediyne chromophore. 


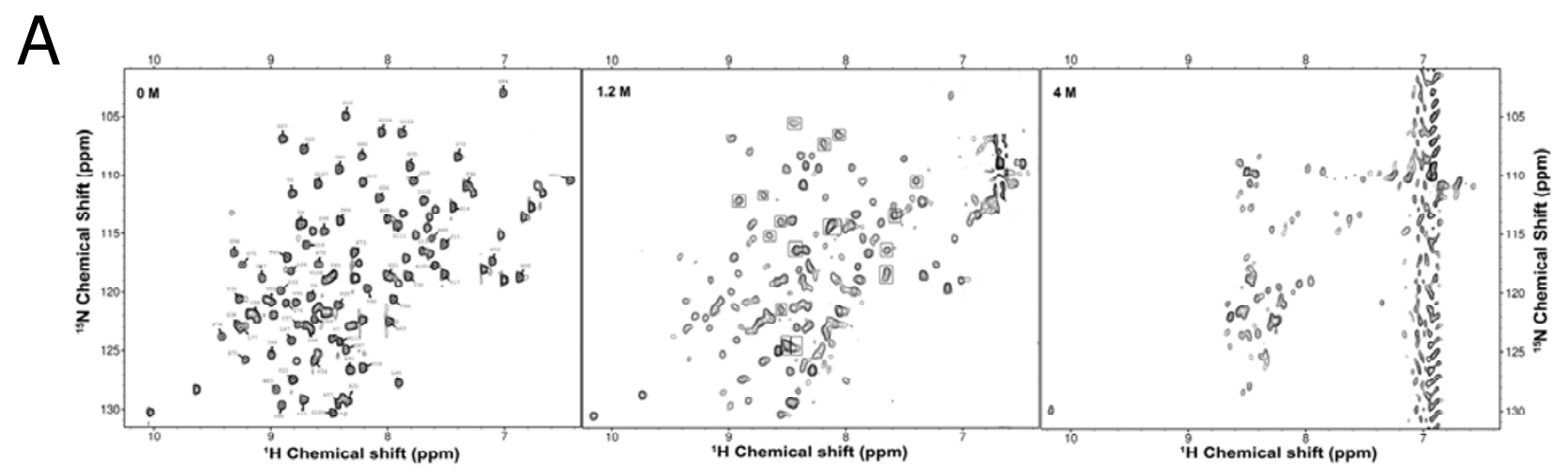

B

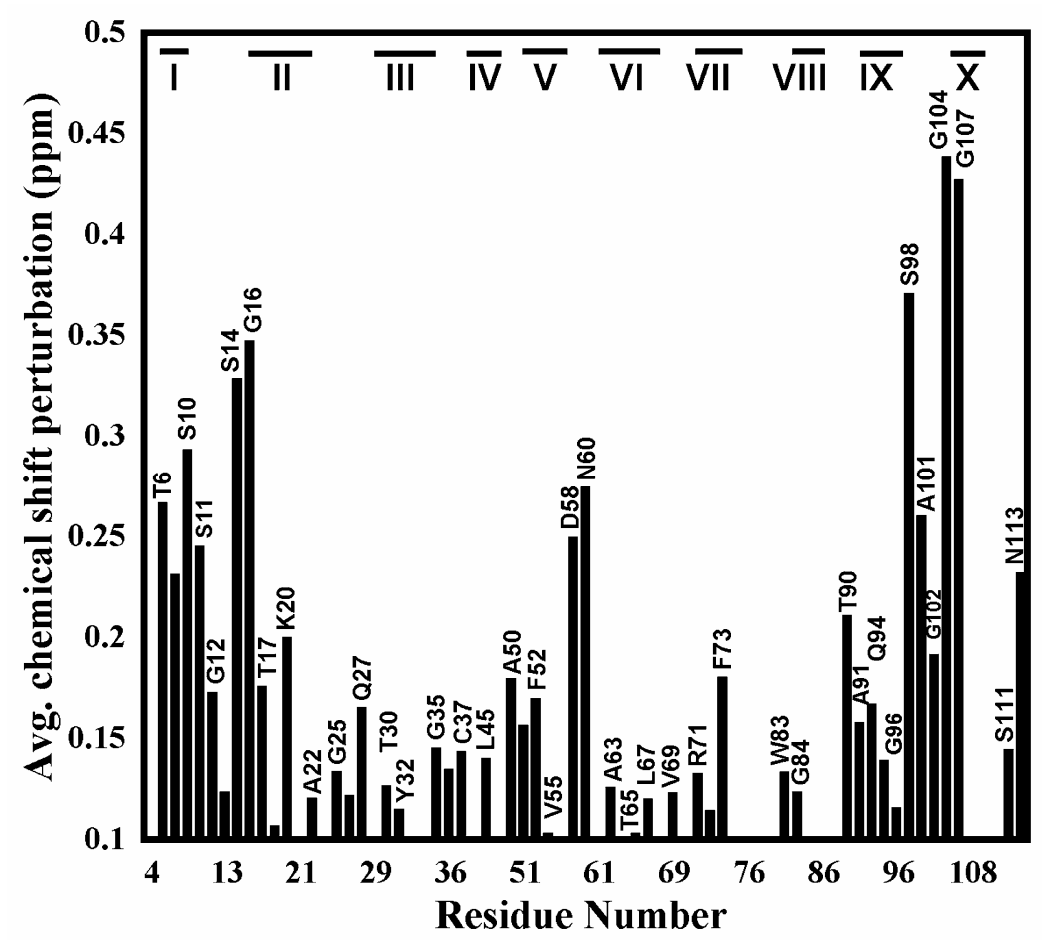

Figure 6

NMR analysis of apoNCS in various concentrations of $\mathbf{G d n H C l}$. (A) ${ }^{1} \mathrm{H}-{ }^{15} \mathrm{~N}$ HSQC spectra of apoNCS obtained at $25^{\circ} \mathrm{C}$ in $0,1.2$, and $4 \mathrm{M} \mathrm{GdnHCl}$. Residues which show maximum perturbation in the intermediate state in $1.2 \mathrm{M} \mathrm{GdnHCl}$ are boxed. (B) The weighted average (of ${ }^{15} \mathrm{~N}$ and ${ }^{\mathrm{H}} \mathrm{H}$ ) chemical shift perturbation of residues in I.2 $\mathrm{M} \mathrm{GdnHCl}$. ApoNCS samples $(720 \mu \mathrm{M})$ were prepared in $10 \mathrm{mM}$ phosphate buffer $(\mathrm{pH} 3)$ in $90 \% \mathrm{H}_{2} \mathrm{O}$ and $10 \% \mathrm{D}_{2} \mathrm{O}$. The $\beta$-strands in the protein are shown in roman numerals (see Fig. I). 
Table I: Protection of NCS-Chr against degradation by binding with MG-like state of apoNCS

\begin{tabular}{|c|c|}
\hline Sample contents & Remaining \% of NCS-Chr ${ }^{a}$ \\
\hline NCS-Chr & $0 \%$ \\
\hline $\mathrm{NCS}-\mathrm{Chr}+1.2 \mathrm{M} \mathrm{GdnHCl}$ & $13 \pm 4 \% b$ \\
\hline holoNCS (NCS-Chr + I:I folded state of apoNCS) & $100 \pm 4 \%$ \\
\hline NCS-Chr + I:I MG-like state of apoNCS (in I.2 M GdnHCl) & $101 \pm 4 \% c$ \\
\hline \multicolumn{2}{|c|}{$\begin{array}{l}\text { a Samples containing } 20 \mu \mathrm{M} \text { NCS-Chr were incubated with folded or MG-like state of apoNCS in } 10 \text { mM phosphate aqueous buffer at pH } 3 \text { and } \\
25^{\circ} \mathrm{C} \text { for } 30 \text { min. Remaining amount of intact NCS-Chr was analyzed by HPLC. } \\
\text { b Most NCS-Chr was degraded in acidic aqueous condition in the presence of I.2 M GdnHCl. The small remaining amount of the NCS-Chr } \\
\text { exhibited additional forms. About } 35 \pm 10 \% \text { of the remaining NCS-Chr was converted into chlorohydrin form of the chromophore. It is known that } \\
\text { the chlorohydrin derivative of NCS-Chr can be obtained by treatment of } \mathrm{HCl} \text { [2]. Presumably, excess amount of GdnHCl triggers the conversion } \\
\text { process. } \\
\text { cNo chlorohydrin conversion could be found in the remaining NCS-Chr in the presence of MG-like state of apoNCS. Presumably, binding of } \\
\text { apoNCS prevents the chromophore conversion reaction triggered by excess amount of GdnHCl. }\end{array}$} \\
\hline
\end{tabular}

\section{MG-like state of apoNCS resumes its folded state after binding to NCS-Chr}

Titration of NCS-Chr into native state apoNCS can produce drastic changes in the near-UV CD spectrum [19,32]. Binding of 1:1 molar ratio of NCS-Chr with native apoNCS produces a prominent negative ellipticity peak minimum at $255 \mathrm{~nm}$, which is a characteristic feature of the native form of holoNCS. Interestingly, titration of 1:1 molar ratio of NCS-Chr into MG-like state of apoNCS in 1.2 $\mathrm{M} \mathrm{GdnHCl}$ at $\mathrm{pH} 3$ produces identical CD spectrum to that of the native holoNCS (Fig. 7). The results unambiguously suggest that NCS-Chr not only can bind to apoNCS in partially unfolded MG-like state but also help the protein fold back into its native state. Without NCSChr, the near-UV CD spectroscopy of the MG-like state of apoNCS shows that $60 \%$ of the tertiary structure is disrupted (Fig. 7 , inset). The observation is consistent with that from intrinsic tryptophan fluorescence study (Fig. 2).

\section{Discussion}

Understanding the mechanism by which a protein folds from denatured state into its unique native three-dimensional structure is an important problem in molecular biology $[33,34]$. ApoNCS, being a model for small all $\beta$ sheet proteins, has been extensively investigated for its folding/unfolding pathways [6-11]. Formation of intermediate state has been suggested when apoNCS is treated with $45 \%$ trifluoroethanol at pH 5 [11]. Under aqueous condition, we demonstrated that the thermal unfolding of apoNCS at pH 7 follows a two-state mechanism [7]. On the contrary, small-angle neutron scattering studies on apoNCS at pH 7 reveal that there could be several discrete intermediate species at equilibrium populated in the unfolding pathways $[9,10]$. The distribution of those substates shows various degrees of residual structures and appears to be temperature or solvent dependent. The major species present during the transition could be considerably unstructured, which may make the thermal unfolding transition look like a two-state transition [9].
This accounts for the failure of identifying a well-defined intermediate state in the unfolding pathway of apoNCS at $\mathrm{pH} 7$ [10]. In the present study, we identified and characterized a stable MG-like state in the GdnHCl-induced unfolding pathway of apoNCS only at a rather acidic $\mathrm{pH}$ (pH 3). At pH 5, where apoNCS is stable at room temperature [7], we have shown that apoNCS is highly resistant against denaturants [35]. The transitions of apoNCS induced by $\mathrm{GdnHCl}$ can not be complete even at the highest concentration of the denaturant. Here we also screened GdnHCl-induced unfolding pathways of aqueous apoNCS at $25^{\circ} \mathrm{C}$ over a $\mathrm{pH}$ range of 3 to 9 (data not shown). We could not observe any stable intermediates except at $\mathrm{pH} 3$. Conceivably, the chances of characterizing a stable intermediate are limited by conditions. We wish the present characterization of a MG-like state intermediate would provide some inputs for further understanding of the complex folding/unfolding mechanism of apoNCS.

Potent anti-neoplastic activity of NCS comes from its NCS-Chr, and apoNCS serves its functional role as a carrier and protector $[1,3,4]$. Without apoNCS, NCS-Chr is very labile and can be inactivated quickly by bases, light, heat, and chemicals such as cellular thiols $[1,3,4]$. When NCS-Chr and apoNCS are biosynthesized from specific gene clusters that produce NCS, the peptide chain of apoNCS needs to fold properly and efficiently to form specific binding cleft for accommodation of the chromophore. Statistical studies have suggested that proteins with more complex topologies such as $\beta$-sheets usually fold more slowly than proteins with $\alpha$-helices [36]. ApoNCS being an all $\beta$-sheet protein, its topology is not favorable for fast folding. Although the role of ligand in protein folding is not well understood, there are studies showing that binding of a ligand prior to protein folding can significantly accelerate the formation of functional protein [37]. Our in vitro experimental data show that apoNCS in its partially unfolded MG-like state resumes its native state after binding with NCS-Chr (Fig. 7). In our opinion, it 


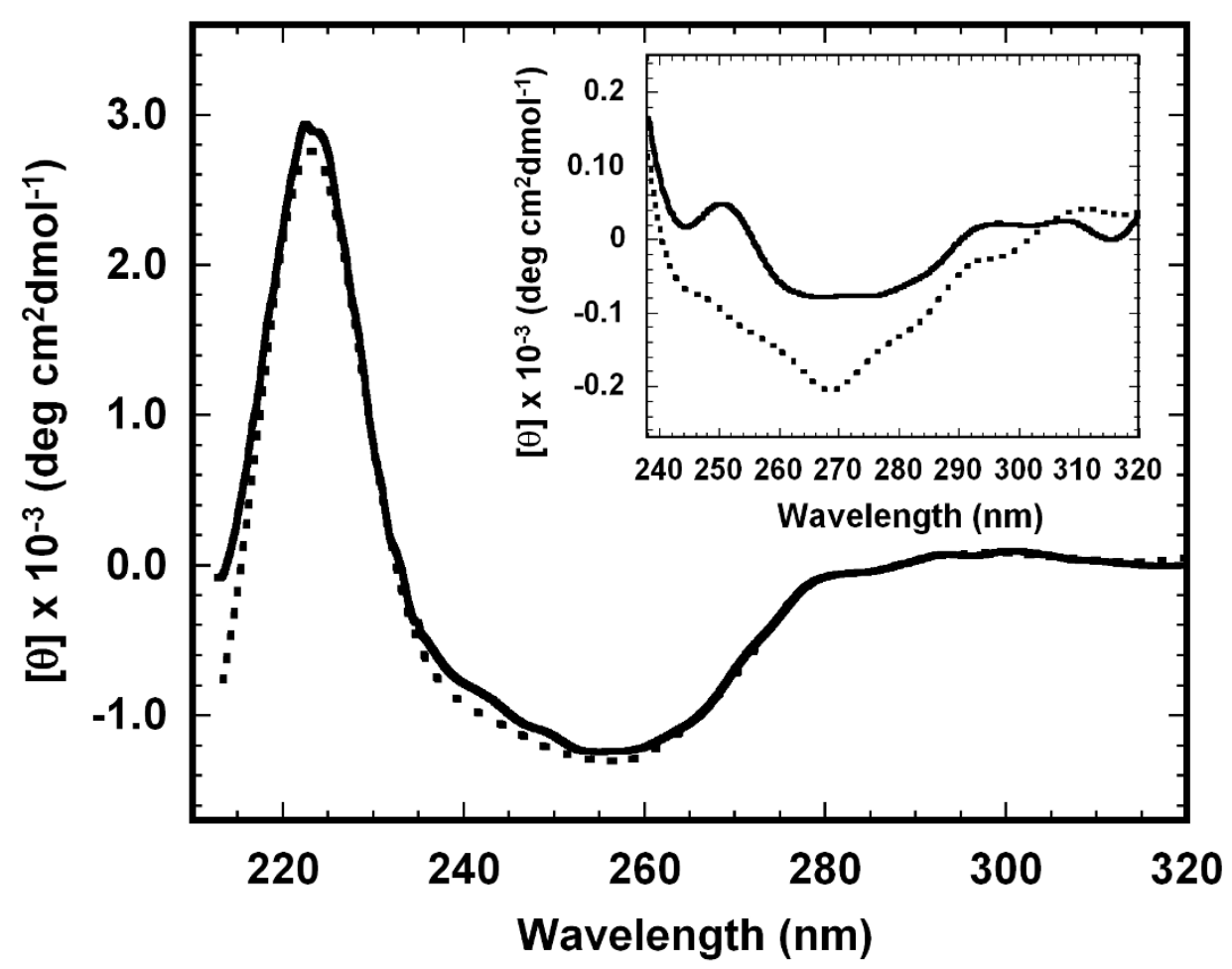

Figure 7

CD spectra of apo- and holoNCS in MG-like and native state. CD spectra of the natural holoNCS (dotted line) in I0 $\mathrm{mM}$ phosphate buffer ( $\mathrm{pH} 3$ ) and the constituted holoNCS of NCS-Chr and MG-like state of apoNCS (full line) in I.2 M Gdn$\mathrm{HCl}(\mathrm{pH} 3)$. MG-like state of apoNCS for constitution was prepared by incubating I $5 \mu \mathrm{M}$ apoNCS with I.2 M GdnHCl in I0 $\mathrm{mM}$ phosphate $(\mathrm{pH} 3)$ at $25^{\circ} \mathrm{C}$ for $30 \mathrm{~min}$. Constitution was performed prior to $\mathrm{CD}$ measurement by adding I:I molar ratio of methanol extracted NCS-Chr into aqueous solution containing MG-like state of apoNCS. Final methanol content was kept minimal to about 4\% (v/v).Inset: Near-UV CD spectrum of apoNCS in MG-like state (full line) (in the presence of I.2 M Gdn$\mathrm{HCl}(\mathrm{pH} 3)$ ) and in folded state (dotted line) (in the absence of $\mathrm{GdnHCl}$ in $10 \mathrm{mM}$ phosphate buffer (pH 3)). All CD spectra were recorded in mean residue ellipticity at $25^{\circ} \mathrm{C}$.

may not be far-fetched to assume that in cellular environment, NCS-Chr binds apoNCS and effectively converts it into a functional protein for its own protection.

Recently, we have demonstrated apoNCS as a superior drug carrier, as its conformation is stable at wide $\mathrm{pH}$ range between 4-10 [7] and is highly resistant against organic solvents and chemical denaturants [35]. Here we further confirmed the superiority of apoNCS, as it exhibits high capability in binding and protecting the labile enediyne chromophore even under harsh acidic environment, where apoNCS conformation becomes intrinsically unstable and disrupted. How is NCS-Chr protected by the apoNCS unfolding intermediate is an interesting question. Based on the structural information obtained from NMR results (Fig. 6), residues T6, S10, S11, S14, and G16 at $\mathrm{N}$-terminus, $\mathrm{N} 60$ and $\mathrm{D} 58$ at the loop region between the $\beta$-strand V (residues 53-57) and VI (residues 62-66), and S98, G104, G107, N113, and S111 at C-terminus are highly perturbed in the MG-like state of apoNCS. On the other hand, majority of the residues involved in the secondary structural interactions do not show appreciable chemical shift changes or broadening. Residues at the bottom of the chromophore binding cleft such as V34, G35, Q36, L45, G96, V95, and V108 show only small perturbation. In addition, the chemical shift of $\mathrm{F} 52$ and the disulfide bond C37-C47, both locate right below the ninemembered enediyne ring, are not significantly affected ( $\Delta \delta$ of $C 47$ is $0.094537 \mathrm{ppm}$, smaller than the $0.1 \mathrm{ppm}$ threshold). It appears that many residues involved in the chromophore binding are not highly perturbed in the transition to the MG-like state. This probably accounts, at least in part, for the retaining ability of the MG-like state of apoNCS in binding and protecting the labile enediyne chromophore.

Besides being potent carrier of the natural ligand enediyne chromophore, apoNCS has also been demonstrated as a 
carrier of small synthetic molecules like EtBr [38], naphthoate ester derivatives $[30,39,40]$ and flavone-based ligand [41]. Interestingly, apoNCS is useful in improving the stability of potent DNA alkylating agents, nitrogen mustards [40]. Furthermore, in vitro 'evolution' studies revealed that apoNCS could be engineered into a common drug delivery vehicle [42]. Drug packaging for drug delivery systems has drawn extensive interests lately in the field of medicinal chemistry. Our study brings insight into the conserving mechanism of naturally occurring NCS and consequently merits apoNCS as a naturally built superior drug carrier for rational drug design strategies.

\section{Conclusion}

We identified and characterized the stable MG-like state accumulated in the equilibrium early unfolding pathway of apoNCS in the presence of $1.2 \mathrm{M} \mathrm{GdnHCl}$ under acidic (pH 3) and aqueous conditions. The apoNCS intermediate retains about $80 \%$ and $40 \%$ of the secondary and tertiary structure, respectively. With the impaired binding cleft, the MG-like state of apoNCS still exhibits full capability of protecting the labile enediyne chromophore. The results demonstrate apoNCS as the natural built-in superior drug carrier. Further CD analyses showed that NCSChr not only binds to the MG-like state of apoNCS but also converts the partially unfolded protein to its functional native state of holoNCS for self protection.

\section{Abbreviations}

NCS: neocarzinostatin; holoNCS: holoneocarzinostatin; apoNCS: aponeocarzinostatin; NCS-Chr: NCS chromophore; GdnHCl: guanidine hydrochloride; ANS: 1-anilino-8-napthalene sulfonate; MG: molten globule; $\mathrm{CD}$ : circular dichroism; $\mathrm{C}_{\mathrm{m}}$ : melting concentration; $\mathrm{T}_{\mathrm{m}}$ : melting temperature; SEC: size-exclusion chromatography; FPLC: fast protein liquid chromatography; HPLC: high pressure liquid chromatography; HSQC: heteronuclear single quantum coherence.

\section{Competing interests}

The authors declare that they have no competing interests.

\section{Authors' contributions}

AS carried out the research project and performed most of the study. TKSK conceived of the work, participated in the design of the study, interpreted the data and helped to draft the manuscript. CMH carried out HPLC and CD analyses for the drug protecting and binding experiments. CY supervised the project, helped in coordination, and provided essential instructions and directions. DHC participated in the design of the study, interpreted and critically reviewed the data, and made revision of the manuscript. All authors read and approved the final manuscript.

\section{Acknowledgements}

This work was supported by a Laboratory Grant (to D.-H. Chin) (NHRIEX90-8807BL) from National Health Research Institutes, and Individual Grants (to D.-H. Chin) (93-2320-B-005-0I2, 93-2II3-M-005-0II) from National Science Council, Taiwan, Republic of China. The work was also supported in part by National Chung Hsing University under the ATU plan funded by the Ministry of Education, Taiwan, Republic of China (to D.-H. Chin). C. Yu was supported by the National Science Council, Taiwan, Republic of China, grants (NSC94-2 I I3-M-007-036, NSC94-2320-B-007005). TKSK was supported by research grants from National Institutes of Health Grants (NIH NCRR COBRE Grant I P20 RR I5569), Department of Energy (DE-FG02-0IERI5I6) and Arkansas Bioscience Institute, Fayetteville, Arkansas, USA. NMR experiments were carried out at the Regional Instrument Center, Department of Chemistry, National Tsing Hua University, Hsinchu, funded by National Science Council, Taiwan, Republic of China. We thank Mr. Ichiro Toishi, Kayaku Co., Ltd., for the supply of NCS powder. We thank Dr. P. Vijaya Palani for proofreading the draft and Dr. Parameswaran Hariharan for examining binding data.

\section{References}

I. Goldberg IH: Mechanism of neocarzinostatin action: Role of DNA microstructure in determination of chemistry of bistranded oxidative damage. Acc Chem Res 1991, 24:191-198.

2. Edo K, Mizugaki M, Koide Y, Seto H, Furihata K, Otake N, Ishida N: The structure of neocarzinostatin chromophore possessing a novel bicyclo[7,3,0]dodecadiyne system. Tetrahedron Lett 1985, 26:33I-334.

3. Kappen LS, Napier MA, Goldberg IH: Roles of chromophore and apo-protein in neocarzinostatin action. Proc Natl Acad Sci U S A 1980, 77:1970-1974.

4. Povirk LF, Goldberg IH: Binding of the nonprotein chromophore of neocarzinostatin to deoxyribonucleic acid. Biochemistry 1980, 19:4773-4780.

5. Chin D-H: Rejection by neocarzinostatin protein through charges rather than sizes. Chem-Eur J 1999, 5:1084-1090.

6. Takashima H, Yoshida T, Ishino T, Hasuda K, Ohkubo T, Kobayashi Y: Solution NMR structure investigation for releasing mechanism of neocarzinostatin chromophore from the holoprotein. J Biol Chem 2005, 280: I I340-I I 346.

7. Jayachithra K, Kumar TKS, Lu T-J, Yu C, Chin D-H: Cold instability of aponeocarzinostatin and its stabilization by labile chromophore. Biophys J 2005, 88:4252-426I.

8. Russo D, Perez J, Zanotti JM, Desmadril M, Durand D: Dynamic transition associated with the thermal denaturation of a small beta protein. Biophys J 2002, 83:2792-2800.

9. Perez J, Vachette P, Russo D, Desmadril M, Durand D: Heatinduced unfolding of neocarzinostatin, a small all-beta protein investigated by small-angle X-ray scattering. J Mol Biol 200I, 308:72I-743.

10. Russo D, Durand D, Calmettes P, Desmadril M: Characterization of the denatured states distribution of neocarzinostatin by small-angle neutron scattering and differential scanning calorimetry. Biochemistry 200I, 40:3958-3966.

II. Sudhahar GC, Balamurugan K, Chin D-H: Release of the neocarzinostatin chromophore from the holoprotein does not require major conformational change of the tertiary and secondary structures induced by trifluoroethanol. I Biol Chem 2000, 275:39900-39906.

12. Sridevi K, Lakshmikanth GS, Krishnamoorthy G, Udgaonkar JB: Increasing stability reduces conformational heterogeneity in a protein folding intermediate ensemble. J Mol Biol 2004, 337:699-7II.

13. Nakao M, Maki K, Arai M, Koshiba T, Nitta K, Kuwajima K: Characterization of kinetic folding intermediates of recombinant canine milk lysozyme by stopped-flow circular dichroism. Biochemistry 2005, 44:6685-6692.

14. Srimathi T, Kumar TKS, Chi Y-H, Chiu I-M, Yu C: Characterization of the structure and dynamics of a near-native equilibrium intermediate in the unfolding pathway of an all beta-barrel protein. J Biol Chem 2002, 277:47507-47516. 
15. Hsieh H-C, Kumar TKS, Chiu C-C, Yu C: Equilibrium unfolding of an oligomeric protein involves formation of a multimeric intermediate state(s). Biochem Biophys Res Commun 2005, 326: $108-114$.

16. Arai $M$, Kuwajima $K$ : Role of the molten globule state in protein folding. Adv Protein Chem 2000, 53:209-282.

17. Ptitsyn OB: How the molten globule became. Trends Biochem Sci 1995, 20:376-379.

18. Kuo H-M, Lee Chao P-D, Chin D-H: Delocalized electronic structure of the thiol sulfur substantially prevents nucleic acid damage induced by neocarzinostatin. Biochemistry 2002, 41:897-905.

19. Hariharan P, Liang W, Chou S-H, Chin D-H: A new model for ligand release. Role of side chain in gating the enediyne antibiotic. J Biol Chem 2006, 28 I: I6025-16033.

20. Palmer AG III, Cavanagh J, Wright PE, Rance M: Sensitivity improvement in proton-detected two-dimensional heteronuclear correlation NMR spectroscopy. J Magn Reson 1991, 93:15I-170.

21. Kay L, Keifer P, Saarinen T: Pure absorption gradient enhanced heteronuclear single quantum correlation spectroscopy with improved sensitivity. J Am Chem Soc 1992, I I 4: 10663-10665.

22. Shaka AJ, Barker PB, Freeman R: Computer-optimized decoupling scheme for wideband applications and low-level operation. J Magn Reson 1985, 64:547-552.

23. Chin D-H, Tseng M-C, Chuang T-C, Hong M-C: Chromatographic and spectroscopic assignment of thiol induced cycloaromatizations of enediyne in neocarzinostatin. Biochim Biophys Acto 1997, I 336:43-50.

24. Heyd B, Lerat G, Adjadj E, Minard P, Desmadril M: Reinvestigation of the proteolytic activity of neocarzinostatin. J Bacteriol 2000, 182: $1812-1818$.

25. Uversky VN: Use of fast protein size-exclusion liquid chromatography to study the unfolding of proteins which denature through the molten globule. Biochemistry 1993, 32:13288-13298.

26. Uversky VN, Semisotnov GV, Pain RH, Ptitsyn OB: 'All-or-none' mechanism of the molten globule unfolding. FEBS Lett 1992 3 | 4:89-92.

27. Buchanan SK: b-Barrel proteins from bacterial outer membranes: structure, function and refolding. Curr Opin Struct Biol | 999, 9:455-46|.

28. Semisotnov GV, Rodionova NA, Razgulyaev OI, Uversky VN, Gripas AF, Gilmanshin RI: Study of the "molten globule" intermediate state in protein folding by a hydrophobic fluorescent probe. Biopolymers |991, 3 I: I| $9-128$

29. Schonbrunner N, Pappenberger G, Scharf M, Engels J, Kiefhaber T: Effect of preformed correct tertiary interactions on rapid two-state tendamistat folding: evidence for hairpins as initiation sites for beta-sheet formation. Biochemistry 1997, 36:9057-9065.

30. Urbaniak MD, Muskett FW, Finucane MD, Caddick S, Woolfson DN: Solution structure of a novel chromoprotein derived from apo-neocarzinostatin and a synthetic chromophore. Biochemistry 2002, 4I:II73I-II739.

31. Povirk LF, Dattagupta N, Warf BC, Goldberg IH: Neocarzinostatin chromophore binds to deoxyribonucleic acid by intercalation. Biochemistry 198I, 20:4007-40I4

32. Napier MA, Kappen LS, Goldberg IH: Effect of nonprotein chromophore removal on neocarzinostatin action. Biochemistry 1980, 19:1767-1773.

33. Nishimura C, Lietzow MA, Dyson HJ, Wright PE: Sequence determinants of a protein folding pathway. J Mol Biol 2005, 35 I:383-392.

34. Roy M, Chavez LL, Finke JM, Heidary DK, Onuchic JN, Jennings PA The native energy landscape for interleukin- I beta. Modulation of the population ensemble through native-state topology. I Mol Biol 2005, 348:335-347.

35. Sudhahar CG, Chin D-H: Aponeocarzinostatin - A superior drug carrier exhibiting unusually high endurance against denaturants. Bioorg Med Chem 2006, 14:3543-3552.

36. Plaxco KW, Simons KT, Baker D: Contact order, transition state placement and the refolding rates of single domain proteins. J Mol Biol 1998, 277:985-994.

37. Wittung-Stafshede P: Role of cofactors in protein folding. Acc Chem Res 2002, 35:201-208.
38. Mohanty S, Sieker LC, Drobny GP: Sequential 'H NMR assignment of the complex of aponeocarzinostatin with ethidium bromide and investigation of protein-drug interactions in the chromophore binding site. Biochemistry 1994, 33:10579-10590.

39. Urbaniak MD, Frost LM, Bingham JP, Kelland LR, Hartley JA, Woolfson DN, Caddick S: Chemical synthesis and cytotoxicity of dihydroxylated cyclopentenone analogues of neocarzinostatin chromophore. Bioorg Med Chem Lett 2003, 13:2025-2027.

40. Urbaniak MD, Bingham JP, Hartley JA, Woolfson DN, Caddick S: Design and synthesis of a nitrogen mustard derivative stabilized by apo-neocarzinostatin. J Med Chem 2004, 47:47I0-47I5.

4I. Caddick S, Muskett FW, Stoneman RG, Woolfson DN: Synthetic ligands for apo-neocarzinostatin. J Am Chem Soc 2006, I 28:4204-4205.

42. Heyd B, Pecorari F, Collinet B, Adjadj E, Desmadril M, Minard P: In vitro evolution of the binding specificity of neocarzinostatin, an enediyne-binding chromoprotein. Biochemistry 2003, 42:5674-5683.
Publish with Bio Med Central and every scientist can read your work free of charge

"BioMed Central will be the most significant development for disseminating the results of biomedical research in our lifetime. "

Sir Paul Nurse, Cancer Research UK

Your research papers will be:

- available free of charge to the entire biomedical community

- peer reviewed and published immediately upon acceptance

- cited in PubMed and archived on PubMed Central

- yours - you keep the copyright 